\title{
Test de caminata de 6 minutos en pacientes de rehabilitación cardiaca de altitud moderada
}

\author{
Nelson F. González*, Claudia V. Anchique y Andrea D. Rivas
}

Grupo de Investigación Mediagnostica Tecmedi S.A.S, Duitama, Colombia

Recibido el 2 de septiembre de 2016; aceptado el 23 de enero de 2017

Disponible en Internet el 22 de marzo de 2017

\author{
PALABRAS CLAVE \\ Enfermedad \\ cardiovascular; \\ Rehabilitación; \\ Test; \\ Ejercicio; \\ Hipoxia
}

\begin{abstract}
Resumen
Introducción: en rehabilitación cardiaca, el test de caminata de 6 minutos es una herramienta eficaz y segura en la evaluación del estado funcional, la eficacia terapéutica, la orientación de modalidades de entrenamiento y el establecimiento del pronóstico de morbilidad y mortalidad. Objetivo: caracterizar la respuesta fisiológica en la caminata de 6 minutos de pacientes en rehabilitación cardiovascular ubicados en altitud moderada, 2.550 metros sobre el nivel del mar, y determinar la importancia clínica.

Métodos: estudio descriptivo de 487 caminatas de 6 minutos en pacientes con edades entre los 18 a 80 años, adscritos a un programa de rehabilitación cardiaca ubicado en altitud moderada. Resultados: los pacientes cardiovasculares colombianos presentan menores distancias recorridas que las demostradas en otras poblaciones y patologías. Durante el test la frecuencia cardiaca se incrementa en 40 latidos por minuto y alcanza el $65 \%$ de la frecuencia cardiaca máxima, en tanto que la presión arterial sistólica aumenta $20 \mathrm{~mm} \mathrm{Hg}$, la percepción del esfuerzo central y periférico aumentan hasta 4 puntos en escala de Borg y disminuye la saturación de oxígeno de 3 puntos porcentuales.

Conclusión: el test caminata de 6 minutos es seguro y bien tolerado en pacientes cardiovasculares ubicados en altitud moderada. Durante la caminata se evidencia cambios significativos en cuanto a respuesta cronotrópica, presora, percepción del esfuerzo y saturación arterial de oxígeno que difieren de resultados encontrados a nivel del mar, fenómeno que aporta valores de referencia para las pruebas realizadas en pacientes cardiovasculares de altitud moderada y para el abordaje clínico de la evaluación de la capacidad funcional, independencia física, riesgo de caídas, ajustes en medicación y valoración integral de comorbilidades.

(c) 2017 Sociedad Colombiana de Cardiología y Cirugía Cardiovascular. Publicado por Elsevier España, S.L.U. Este es un artículo Open Access bajo la licencia CC BY-NC-ND (http:// creativecommons.org/licenses/by-nc-nd/4.0/).
\end{abstract}

\footnotetext{
* Autor para correspondencia.

Correo electrónico: nelsonfgonzalezc@gmail.com (N.F. González).
} 


\section{KEYWORDS}

Cardiovascular

disease;

Rehabilitation;

Test;

Exercise;

Hypoxia

\section{Six-minute walk test in cardiac rehabilitation patients at moderate altitude in Colombia}

\begin{abstract}
Introduction: The 6-minute walk test in cardiac rehabilitation is and effective and safe tool for the evaluation of functional status, as well of the effectiveness of the therapy, the adjustment of training methods, and to establish the morbidity and mortality prognosis.

Objective: To investigate the aspects that affect the physiological response in the 6-minute walk test in cardiovascular rehabilitation patients at moderate altitudes $(2,550$ metres above sea (evel), and determine the clinical importance.

Material and methods: A descriptive study was conducted on 487 six-minute walks by patients with ages between 18 and 80 years, enrolled in cardiac rehabilitation programme and who lived at a moderate altitude.

Results: The Colombian cardiovascular patients covered shorter distances than those demonstrated in other populations and other diseases. The heart rate during the test increased by 40 beats per minute and reached $65 \%$ of the maximum heart rate, as such that the systolic blood pressure increased by $20 \mathrm{mmHg}$. The perception of central and peripheral effort also increased up to 4 points on the Borg scale, as well as a mean decrease of $3 \%$ in oxygen saturation.

Conclusion: The 6-minute test is safe and well-tolerated in cardiovascular patients living at moderate altitudes. Significant changes were seen during the walk as regards chronotropic response, pressure, perception of effort, oxygen saturation levels, which were different from the results found at sea level. This phenomenon provides reference values for tests performed on cardiovascular patients at moderate altitudes, as well as for the clinical approach to the evaluation of the functional capacity, physical independence, falls risk, adjustments to medication, and an integral assessment of comorbidities.

(c) 2017 Sociedad Colombiana de Cardiología y Cirugía Cardiovascular. Published by Elsevier España, S.L.U. This is an open access article under the CC BY-NC-ND license (http:// creativecommons.org/licenses/by-nc-nd/4.0/).
\end{abstract}

\section{Introducción}

El test caminata de seis minutos (TC6M) fue descrito por Guyatt et al. ${ }^{1,2}$. Es una prueba de campo que inicialmente demostró utilidad en la evaluación de la tolerancia al ejercicio físico en pacientes con patología respiratoria crónica ${ }^{3}$, y posteriormente se utilizó para evaluar el impacto terapéutico en pacientes con falla cardiaca, para convertirse hoy en una herramienta eficaz en la evaluación de la capacidad funcional y el pronóstico de morbimortalidad en el paciente cardiovascular ${ }^{4}$.

Uno de los componentes de la rehabilitación cardiaca es el test ergométrico, el cual se usa para cuantificar tolerancia al esfuerzo, especificar estado funcional, evaluar eficacia terapéutica y orientar modalidades de entrenamiento ${ }^{5}$. La ergo-espirometría proporciona toda esta información ${ }^{6,7}$ y sigue siendo el "estándar de oro" en este objetivo, sin embargo, requiere recursos humanos y técnicos que aumentan los costos en la práctica clínica habitual y puede no ser representativa de las actividades de la vida real diaria ${ }^{8}$. En consecuencia, el test de caminata con medición de parámetros fisiológicos emerge como alternativa eficaz y segura, reflejando una actividad humana cotidiana ${ }^{9,10}$.

El TC6 $\mathrm{M}$ es una prueba cardiopulmonar validada ${ }^{11}$, de bajo costo, no invasiva, reproducible, bien tolerada ${ }^{12}$, en su mayoría de intensidad submáxima y aproximadamente equivalente al primer umbral ventilatorio ${ }^{13}$. Permite evaluar en forma global no sólo la respuesta de los sistemas cardiovascular y pulmonar, sino de todos los demás sistemas involucrados con el intercambio gaseoso ${ }^{14}$. El resultado de la prueba generalmente se cita como el número de metros cubiertos en 6 minutos y el porcentaje correspondiente de un predicho teórico calculado teniendo en cuenta la edad y los parámetros antropométricos del paciente ${ }^{15}$.

Para su interpretación correcta se requieren los valores de referencia de la población en la que se efectúa la prueba. En Colombia la falta de valores de referencia obliga a utilizar valores obtenidos en estudios extranjeros ${ }^{14-17}$, que tal vez pudieran no reflejar las condiciones propias de pacientes cardiovasculares colombianos con exposición a hipoxia por altura; de hecho, recientemente se ha demostrado que las ecuaciones extranjeras no son adecuadas para poblaciones brasileñas, chilenas, tunecinas entre otras ${ }^{18-25}$. También es importante reconocer que la disminución de la presión de oxígeno por una altitud moderada puede no afectar las funciones de un sujeto en reposo, pero sí la respuesta fisiológica y el rendimiento en ejercicio de intensidad moderada y alta ${ }^{26}$.

El propósito de este estudio ha sido caracterizar y analizar la respuesta fisiológica durante el TC6 M en una población de pacientes cardiovasculares ubicados en altitud de $\mathbf{2 . 5 5 0}$ metros sobre el nivel del mar (msnm) en Colombia, con miras a determinar su importancia clínica y generar valores de referencia que ayuden en el abordaje la prueba. 


\section{Materiales y métodos}

Se llevó a cabo un estudio observacional descriptivo que incluye 487 test de caminata de 6 minutos en pacientes que asistieron al programa de rehabilitación cardiaca en el centro de cardiología de Mediagnostica Tecmedi ubicado a 2.550 msnm en Duitama (Boyacá, Colombia) durante el transcurso de febrero de 2014 a agosto de 2015. El protocolo utilizado para la aplicación del TC6 M es el recomendado por la Sociedad Americana de Tórax ${ }^{17}$.

Se incluyeron pacientes con indicación de rehabilitación cardiaca y se excluyeron aquellos con angina inestable, frecuencia cardiaca basal $\geq 120$ latidos por minuto o con presión arterial en reposo mayor o igual $180 / 100 \mathrm{~mm} \mathrm{Hg}$. Todos firmaron el consentimiento informado. Los procedimientos se ajustan a las normas éticas del comité de experimentación humana y a la Asociación Médica Mundial y la Declaración de Helsinki.

La prueba se realizó en un corredor de 30 metros de longitud, ubicado en el interior de la institución prestadora de servicio. Desde el inicio y hasta el final de la prueba se midió la frecuencia cardiaca con cardio-frecuenciómetro (Polar ${ }^{\circledR}$ FT1), mientras que la saturación arterial de oxígeno con un oxímetro de pulso (Nonin Medical Inc., Plymouth, Mn., USA) y la percepción del esfuerzo con escala de Borg modificada.

\section{Análisis estadístico}

En el primer paso se hizo la estadística descriptiva de las variables; las continuas se expresaron en media \pm desviación estándar y mediana, según correspondiera, mientras que las categóricas se exponen en términos de frecuencia. En el segundo paso, para determinar diferencias entre el estado basal y al final de los 6 minutos, se utilizó la prueba $t$ de Student para muestras relacionadas y la prueba de Kruskal-Wallis para las variables que no cumplían con una distribución normal según prueba Shapiro-Wilk. Para todos los análisis se consideraron valores de $\mathrm{p}$ inferiores a 0,05 estadísticamente significativos, mediante software SPSS $^{\oplus} 18$.

\section{Resultados}

En a tabla 1 se muestran la distribución y las características de la población total de estudio y separadas por género. Se evidenció mayor número de participantes masculinos con peso y talla superiores a los del género femenino, pero sin diferencia significativa en el índice de masa corporal ni en la edad entre géneros.
Tabla 1 Distribución y características de la población

\begin{tabular}{llll}
\hline & Total & Mujeres & Hombres \\
\hline & $\mathrm{n}=487$ & $\mathrm{n}=160$ & $\mathrm{n}=327$ \\
Edad (años) & $62,9 \pm 11$ & $61,8 \pm 11$ & $63,5 \pm 10$ \\
Peso $(\mathrm{kg})$ & $71,8 \pm 11$ & $64,6 \pm 10$ & $75,3 \pm 10$ * \\
Talla $(\mathrm{cm})$ & $162,1 \pm 8$ & $153,4 \pm 6$ & $166,4 \pm 6$ \\
IMC $\left(\mathrm{kg} / \mathrm{m}^{2}\right)$ & $27,2 \pm 3,3$ & $27,4 \pm 4$ & $27,1 \pm 3$ \\
\hline
\end{tabular}

IMC: índice de masa corporal, $p<0,05$.

La población de estudio tiene una distribución etaria que incluye sujetos en diferentes décadas de la vida; jóvenes entre 18 y 39 años (8\%), mediana edad $40-64$ años (56\%) y adulto mayor de 65-80 años (36\%). La mayoría de las pruebas se aplicaron a pacientes con diagnóstico de enfermedad coronaria posterior a evento isquémico agudo y con revascularización miocárdica $(71 \%)$; el porcentaje restante de caminatas correspondió a pacientes con diagnóstico de corrección de valvulopatías y cardiopatías congénitas (12\%), falla cardiaca estable y portadores de dispositivos (11\%) y síncope $(5 \%)$. Entre tanto, para los factores de riesgo la proporción en la población total está dada por hipertensión arterial $(63 \%)$, diabetes $(17 \%)$, dislipidemia $(80 \%)$, obesidad $(17 \%)$, sobrepeso $(49 \%)$, obesidad abdominal $(74 \%)$ y sedentarismo (42\%); es importante resaltar que solo el $27 \%$ de la población presentó tres o menos factores de riesgo y que el $94 \%$ consume betabloqueador, condición relevante en la respuesta cardiovascular al ejercicio físico.

El comportamiento de las variables fisiológicas de un sujeto promedio previas a iniciar el TC6M se muestra en la tabla 2, donde no se evidencian diferencias significativas entre hombres y mujeres. Los cambios experimentados durante el TC6M en frecuencia cardiaca, presión arterial, saturación de oxígeno, percepción de esfuerzo, velocidad y capacidad funcional en MET (calculada por formula de Roos $^{14}$ ) para un sujeto promedio y diferenciado por género, se describen en la tabla 3. Los valores en distancia recorrida, delta de frecuencia cardiaca, frecuencia cardiaca máxima, capacidad funcional y velocidad son superiores en hombres respecto a los valores obtenidos en el género femenino. Por ejemplo, la distancia recorrida fue significativamente superior en hombres que en mujeres ( $513,6 \pm 5,6$ vs. $458,7 \pm 6,8$ respectivamente, $p<0,0001$ ).

Cuando se comparó entre los diferentes diagnósticos o indicaciones de rehabilitación cardiaca, como lo muestra la tabla 4, se pudo evidenciar que los pacientes con diagnóstico de falla cardiaca tienen una distancia recorrida promedio al final de los seis minutos, inferior a 40,7 metros comparada

Tabla 2 Parámetros fisiológicos previos al inicio del test de caminata de 6 minutos

\begin{tabular}{|c|c|c|c|c|}
\hline & $\begin{array}{l}\text { FC inicial } \\
\text { (lpm) }\end{array}$ & $\begin{array}{l}\text { PAS inicial } \\
(\mathrm{mm} \mathrm{Hg})\end{array}$ & $\begin{array}{l}\text { PAD inicial } \\
(\mathrm{mm} \mathrm{Hg})\end{array}$ & $\begin{array}{l}\mathrm{SaO}_{2} \\
\text { inicial (\%) }\end{array}$ \\
\hline Total & $66 \pm 10$ & $111 \pm 14$ & $70 \pm 8$ & $94 \pm 2$ \\
\hline Femenino & $66,5 \pm 10$ & $111 \pm 16$ & $69 \pm 8$ & $93,7 \pm 2$ \\
\hline Masculino & $65,9 \pm 10$ & $111 \pm 13$ & $70 \pm 8$ & $94,1 \pm 2$ \\
\hline
\end{tabular}

FC: frecuencia cardiaca, Ipm: latidos por minuto, PAS: presión arterial sistólica, PAD: presión arterial diastólica, SaO ${ }_{2}$ : saturación de oxígeno. 
con las distancias recorridas por pacientes con enfermedad coronaria o con otros diagnósticos (enfermedad coronaria $496,9 \pm 5,18$ vs. falla cardiaca 456,2 $\pm 13,58, p<0,005)$; sin embargo, en las demás variables fisiológicas medidas, no existieron diferencias estadísticamente significativas entre los distintos diagnósticos.

\section{Discusión}

Este estudio proporciona información sobre los cambios en las variables fisiológicas obtenidas en caminatas de seis minutos en pacientes de un programa de rehabilitación cardiovascular, desarrolladas en una altitud de $2.550 \mathrm{msnm}$ en Colombia. Tiene, además, dos características diferenciales; la primera es que cuenta con gran cantidad de pruebas hechas en diferentes patologías cardiovasculares (enfermedad coronaria, falla cardiaca, valvulopatías y síncope), en distintas fases de rehabilitación cardiovascular, con amplia distribución etaria y características morfológicas que concuerdan con los datos publicados en la Encuesta Nacional de la Situación Nutricional - ENSIN 2010²7, de modo que incrementa la validez externa de los resultados y genera confiabilidad para su uso y aplicación en pacientes cardiovasculares en Colombia. La segunda característica está dada por ser una población con sujetos expuestos a hipoxia hipobárica por residir en altitud entre 2.500 a 3.500 msnm, determinante importante en la respuesta cardiopulmonar al ejercicio físico ${ }^{26}$; incluso, esta misma población cuenta con un altísimo porcentaje (94\%) de sujetos bajo el efecto farmacológico de betabloqueador, condición que influye también de forma ostensible en la respuesta cardiopulmonar ${ }^{28}$. Con base en lo anterior este estudio intenta determinar la importancia de estas variables en la ejecución e interpretación del TC6 M, ya que la mayoría de estudios efectuados hasta ahora son a nivel del mar (de 0 a $1.500 \mathrm{msnm}$ ) y se desconocen valores de referencia para poblaciones en altitud y con patología cardiovascular.

El primer hallazgo de importancia en este estudio corresponde a que el TC6M es una prueba de ejercicio bien tolerada y segura para el paciente de rehabilitación cardiovascular en altitud moderada; durante los 18 meses de seguimiento no se evidenció evento adverso durante la caminata, todos los pacientes terminaron el test sin complicaciones, y aunque el objetivo no era determinar la morbimortalidad se pudo confirmar que distancias recorridas inferiores a 300 metros pronostican el requerimiento de un mayor número de hospitalizaciones, así como de probabilidad de muerte, como se ha demostrado en estudios de otras poblaciones del mundo ${ }^{4}$.

Un segundo hallazgo se relaciona con que la distancia recorrida en la caminata de 6 minutos (DRC6M) por pacientes cardiovasculares colombianos, es inferior a la calculada por fórmulas extranjeras; las distancias recorridas son en promedio inferiores en 26,8 metros respecto a las calculadas por fórmula de Enright y Sherril ${ }^{16}$ (realizada en 173 mujeres y 117 hombres norteamericanos), e inferiores en 102,9 metros por fórmula de Troosters et al. ${ }^{17}$ (53 sujetos de ambos géneros en Bélgica). La búsqueda de estudios que incluyeran población colombiana arrojó resultados escasos; entre ellos figura el estudio multicéntrico de Casanova et al. ${ }^{29}$ que incluyó 39 sujetos colombianos y halló una DRC6M de 
Tabla 4 Variables fisiológicas en el test de caminata de 6 minutos según tipo de diagnóstico o indicación de rehabilitación cardiaca

\begin{tabular}{|c|c|c|c|c|c|c|c|}
\hline & $\begin{array}{l}\text { FC inicial } \\
(\mathrm{lpm})\end{array}$ & $\begin{array}{l}\text { Delta en FC } \\
(\mathrm{lpm})\end{array}$ & $\begin{array}{l}\text { Porcentaje } \\
\text { de FC } \\
\text { máxima }\end{array}$ & $\begin{array}{l}\text { Delta PAS } \\
(\mathrm{mm} \mathrm{Hg})\end{array}$ & $\begin{array}{l}\text { Delta de } \\
\mathrm{SaO}_{2}\end{array}$ & $\begin{array}{l}\text { Delta de } \\
\text { Borg }\end{array}$ & $\begin{array}{l}\text { Distancia } \\
\text { recorrida } \\
\text { (m) }\end{array}$ \\
\hline $\begin{array}{c}\text { Enfermedad } \\
\text { coronaria }\end{array}$ & $65,0 \pm 10,5$ & $41,8 \pm 17,1$ & $65,8 \pm 9,7$ & $23,1 \pm 15,0$ & $-2,568 \pm 0,18$ & $2,2 \pm 1,7$ & $496,9 \pm 5,19$ \\
\hline $\begin{array}{l}\text { Congénitas y } \\
\text { valvulopatías }\end{array}$ & $68,7 \pm 11,4$ & $41,7 \pm 18,8$ & $65,6 \pm 9,8$ & $19,2 \pm 13,4$ & $-2,21 \pm 0,47$ & $1,6 \pm 1,4$ & $502,8 \pm 12,17$ \\
\hline $\begin{array}{c}\text { Falla cardiaca y } \\
\text { dispositivos }\end{array}$ & $66,1 \pm 10,8$ & $38,2 \pm 18,2$ & $64,6 \pm 11,1$ & $17,3 \pm 11,4$ & $-3,444 \pm 1,19$ & $2,1 \pm 1,8$ & $456,2 \pm 13,58$ \\
\hline Síncope & $67,8 \pm 8,2$ & $43,6 \pm 16,3$ & $64,7 \pm 9,3$ & $16,9 \pm 15,5$ & $-2,568 \pm 0,18$ & $2,5 \pm 2,0$ & $524,8 \pm 25,3$ \\
\hline
\end{tabular}

$632 \pm 63$ metros, distancia también superior a la encontrada en el presente estudio. Las causas que posiblemente expliquen menores DRC6M en este estudio, están dadas por edades y pesos promedio superiores a los de estudios en comparación, ya la literatura ha demostrado correlación directa entre menor DRC6M y personas mayores, con sobrepeso o con obesidad secundario a una longitud de paso más corta ${ }^{15,18,21}$. Otro condicionante importante que podría explicar una menor DRC6 $\mathrm{M}$ en este estudio es la gran proporción de pacientes con posoperatorio temprano de cirugía cardiaca y estadios avanzados de falla cardiaca; ambas situaciones se asocian con distancias notablemente inferiores como lo demostraron los estudios de Fiorina et al. ${ }^{30}$ con 1.370 pacientes de posoperatorio cardiaco y el de Lipkin et al. ${ }^{31}$ con 56 pacientes con falla cardiaca, encontrando una DRC6M promedio entre 304 a 405 metros. Si se tiene en cuenta la diferencia entre la DRC6 M encontrada en los pacientes de rehabilitación cardiaca en Colombia y la calculada por fórmulas extranjeras, se sugiere diseñar y validar una fórmula de regresión específica para la población colombiana y por el momento elegir la fórmula teórica que cuente con las menores diferencias respecto a los resultados encontrados en este estudio, ya que la elección equívoca de una fórmula de referencia puede traducirse en errores de interpretación del nivel de aptitud física y de seguimiento a intervenciones ${ }^{19,32}$.

Los resultados también confirman que el número de metros recorridos es significativamente superior en hombres que en mujeres, con una diferencia promedio de 54,9 $\pm 9,4$ metros ( $p<0,0001)$. Múltiples estudios en diferentes poblaciones del mundo comparten este hallazgo, el cual obedece a que el género femenino es de talla más baja y tiene una longitud de paso más corta ${ }^{21-25}$.

El tercer hallazgo importante y centro objetivo de investigación, está relacionado con los parámetros de respuesta fisiológica en el TC6M, que a pesar de ser de frecuente medición es difícil encontrar en la literatura valores de referencia que puedan usarse en el abordaje del TC6M de personas sanas y pacientes cardiovasculares. Este estudio proporciona los posibles valores de un sujeto expuesto a una altitud intermedia con patología cardiovascular en reposo previo a iniciar una caminata de 6 minutos y los posibles cambios fisiológicos en frecuencia cardiaca, presión arterial, saturación de oxígeno y percepción de esfuerzo al final del test (tablas 2 y 3), que permiten afrontar el abordaje del TC6 M del paciente cardiovascular colombiano de una forma más dinámica.

En cuanto a la respuesta cronotrópica en el TC6 M, se evidenció que los sujetos alcanzan el 65,6 $\pm 9 \%$ de la frecuencia cardiaca máxima (\%FCM) y un delta de frecuencia cardiaca $(\triangle F C)$ de $42 \pm 17$ lpm, con lo cual se infiere que el TC6M es un test de ejercicio predominantemente submáximo, que genera cambios significativos en la respuesta cronotrópica. Resultados similares fueron reportados por Chetta et al. ${ }^{33}$ con sujetos que alcanzaron el $67 \pm 10 \%$ de la FCM, mientras que investigaciones como la de Osses et al. ${ }^{22}$ hallaron el $74 \pm 12 \%$ y Casanova et al. ${ }^{29}$ con población colombiana encontraron el $88 \pm 10 \%$ de la FCM. En cuanto a la discusión de esta diferencia, la fórmula para calcular la frecuencia cardiaca máxima puede ser diferente; en la presente investigación la fórmula elegida fue Tanaka ${ }^{34}$, y en segundo lugar, como se había enunciado, está el gran porcentaje (94\%) de población que consume betabloqueador, hecho que puede llevar a asumir que pacientes en rehabilitación cardiaca alcanzaran menores porcentajes de frecuencia cardiaca máxima. Es importante tener en cuenta que los betabloqueadores interfieren no sólo en la frecuencia cardíaca final alcanzada en ejercicio sino también con la frecuencia cardiaca en reposo, de modo que en el abordaje del TC6M el \%FCM y el $\triangle F C$ ayudan en la evaluación de la respuesta cronotrópica permitiendo determinar si un paciente se encuentra bien betabloqueado, dado que una frecuencia cardiaca basal baja y un incremento en la frecuencia cardiaca inferior a $60 \mathrm{lpm}$ así lo indican, y en caso contrario el TC6 M permitirá ayudar en la titulación y ajuste de dosis de dichos medicamentos.

Un cuarto hallazgo en esta investigación está en relación con la saturación de oxígeno $\left(\mathrm{SaO}_{2}\right)$, pues se evidenció que en reposo es inferior a la encontrada en estudios hechos a nivel del $\operatorname{mar}^{20,22,33}$. La $\mathrm{SaO}_{2}$ basal promedio en reposo de la población de estudio fue $94 \%$ y estudios realizados a nivel del mar han reportado valores de 96 a $98 \%$. También, en este estudio se evidenció un descenso en la $\mathrm{SaO}_{2}$ hasta de 5 puntos porcentuales durante el test, hallazgo que tampoco ha sido evidenciado en investigaciones hechas a nivel del mar. La disminución más marcada en la 
saturación de oxígeno para una población expuesta a hipoxia hipobárica relacionada con altitud moderada, puede explicarse por una disminución en la presión atmosférica y en la disponibilidad de oxígeno que desencadena una $\mathrm{SaO}_{2}$ basal menor y una tendencia a la desaturación durante el ejercicio submáximo ${ }^{26}$. Sin embargo, se sugiere continuar con investigaciones que permitan esclarecer las diferencias en la respuesta fisiológica por la altura, y se propone que saturaciones basales más bajas en reposo y en ejercicio pudieran ser hallazgos no necesariamente patológicos en sujetos expuestos a la altura, como los de este estudio.

El quinto hallazgo está relacionado con la percepción del esfuerzo cuantificado mediante escala de Borg. El TC6M genera sintomatología significativa en el paciente cardiovascular; esta investigación muestra un incremento tanto a nivel central como de fatiga periférica, con cambios entre el inicio y el final de hasta 4 puntos en la escala de Borg. Hallazgos similares encontraron Baeza-Barría et al. ${ }^{35}$ en 30 pacientes chilenos con enfermedad pulmonar crónica, pero no fueron evidenciados en sujetos sanos, hecho que probablemente se justifique en que los pacientes cardiopulmonares presentan disminución en la fuerza y la resistencia secundaria a una baja en la proporción de fibras musculares, así como en la irrigación muscular y en la actividad enzimática aeróbica ${ }^{35}$. Con base en lo anterior, puntuaciones superiores a cuatro puntos en la escala de Borg modificada para percepción central y periférica, sugieren angina o enfermedad arterial periférica respectivamente, y se requerirán estudios complementarios.

Por último, esta investigación encontró que la velocidad promedio alcanzada por los pacientes de rehabilitación cardiaca es de $4,9 \mathrm{~km}$ por hora, similar a la encontrada en un reciente estudio multicéntrico internacional ${ }^{32}$. Esta variable permite determinar la funcionalidad e independencia física de un individuo; estudios han demostrado que una velocidad inferior a $4,4 \mathrm{~km}$ por hora es predictor de alta probabilidad de caídas, dependencia urbana y mal pronóstico de supervivencia $^{36}$. Por tanto, esta variable es relevante dentro del programa de rehabilitación cardiaca para determinar el riesgo de caídas y la aptitud de desplazamiento de los pacientes con o sin compañía dentro y fuera del programa.

\section{Conclusión}

El TC6 M es seguro y bien tolerado por el paciente cardiovascular, pues no se evidenciaron eventos adversos durante su realización. En su mayoría, es un test submáximo que genera un espectro de cambios en la respuesta cardiopulmonar, que va más allá de la sola interpretación de la distancia recorrida en seis minutos, aportando información relevante al clínico en cuanto a evaluación de la capacidad funcional, independencia física, riesgo de caídas, ajustes en la medicación y valoración integral de comorbilidades; así mismo, la evaluación de la respuesta cronotrópica y presora permite evaluar la eficacia terapéutica farmacológica, los cambios en la saturación de oxígeno permiten determinar la necesidad de prescripción de oxígeno suplementario, los cambios en la percepción de esfuerzo permiten hacer seguimiento de comorbilidades como la enfermedad arterial periférica, y los valores de velocidad predicen independencia y riesgo de caídas.
El TC6M se convierte entonces en una herramienta valiosa en la aproximación diagnóstica y de seguimiento terapéutico del paciente cardiovascular; no obstante, los valores encontrados en pacientes de altitud difieren a los encontrados a nivel del mar, característica que sugiere que la adopción de fórmulas extranjeras puede no reflejar la realidad, y por tanto se debe continuar en el diseño de investigaciones que permitan construir y validar valores de referencia específicos para cada población, además de dilucidar el efecto de variables externas como la altitud y otras no incluidas dentro de este estudio.

De acuerdo con la revisión y el conocimiento de los autores, este es el primer estudio que describe las variables fisiológicas del TC6M en pacientes cardiovasculares en altitud moderada y evidencia su utilidad clínica y la necesidad de emprender más estudios que permitan validar valores de referencia específicos para cada población con mira a esclarecer el efecto de variables externas como la altitud.

\section{Responsabilidades éticas}

Protección de personas y animales. Los autores declaran que los procedimientos seguidos se conformaron a las normas éticas del comité de experimentación humana responsable y de acuerdo con la Asociación Médica Mundial y la Declaración de Helsinki.

Confidencialidad de los datos. Los autores declaran que han seguido los protocolos de su centro de trabajo sobre la publicación de datos de pacientes.

Derecho a la privacidad y consentimiento informado. Los autores han obtenido el consentimiento informado de los pacientes y/o sujetos referidos en el artículo. Este documento obra en poder del autor de correspondencia.

\section{Financiación}

Mediagnostica Tecmedi, Duitama, Colombia. Salarios y equipos.

\section{Conflicto de intereses}

Ninguno

\section{Agradecimientos}

Al grupo de pacientes y al equipo paramédico y administrativo de Mediagnostica Tecmedi SAS.

\section{Bibliografía}

1. Guyatt GH, Sullivan MJ, Thompson PJ, Fallen EL, Pugsley SO, Taylor DW, et al. The 6-minute walk: a new measure of exercise capacity in patients with chronic heart failure. Can Med Assoc J. 1985;132:919-23.

2. McGavin CR, Gupta SP, McHardy GRJ. Twelve minute walking test for assessing disability in chronic bronchitis. BMJ. $1976 ; 1: 822-3$. 
3. Butland RJ, Pang J, Gross ER, Woodcock AA, Geddes DM. Two-, six-, and 12-minute walking tests in respiratory disease. Br Med J (Clin Res Ed). 1982;284:1607-8.

4. Papathanasiou JV, Ilieva E, Marinov B. Six-minute walk test: an effective and necessary tool in modern cardiac rehabilitation. Hellenic J Cardiol. 2013;54:126-30.

5. Solway S, Brooks D, Lacasse Y, Thomas S. A qualitative systematic overview of the measurement properties of functional walk tests used in the cardiorespiratory domain. Chest. 2001;119:256-70.

6. Gibbons RJ, Balady GJ, Bricker JT, Chaitman BR, Fletcher GF, Froelicher VF, et al. ACC/AHA 2002 guideline update for exercise testing: summary article. A report of the American College of Cardiology/American Heart Association Task Force on Practice Guidelines (Committee to Update the 1997 Exercise Testing Guidelines). J Am Coll Cardiol. 2002;40:1531-40.

7. Mezzani A, Hamm LF, Jones AM, McBride PE, Moholdt T, Stone $\mathrm{JA}$, et al. Aerobic exercise intensity assessment and prescription in cardiac rehabilitation: a joint position statement of the European association for cardiovascular prevention and rehabilitation, the American association of cardiovascular and pulmonary rehabilitation, and the Canadian association of cardiac rehabilitation. J Cardiopulm Rehabil Prev. 2012;32:327-50.

8. Houghton AR, Harrison M, Cowley AJ, Hampton JR. Assessing exercise capacity, quality of life and haemodynamics in heart failure: do the tests tell usthesamething? Eur J Heart Fail. 2002;4:289-95.

9. Ades PA, Savage P, Cress ME, Brochu M, Lee NM, Poehlman ET. Resistance training on physical performance in disabled older female cardiac patients. Med Sci Sports Exerc. 2003;35:1265-70.

10. Singh S. The use of field walking tests for assessment of functionnal capacity in patients with chronic airways obstruction. Physiotherapy. 1992;78:102-4.

11. Hamilton DM, Haennel RG. Validity and reliability of the 6minute walk test in a cardiac rehabilitation population. J Cardiopulm Rehabil. 2000;20:156-64.

12. Tokmakova M. Cardiopulmonary stress test-general characteristics and clinical value. Folia Med. 1998;3:38-42.

13. Noonan V, Dean E. Submaximal exercise testing: clinical application and interpretation. Phys Ther. 2000;80:782-807.

14. Ross RM, Murthy JN, Wollak ID, Jackson AS. The six minute walk test accurately estimates mean peak oxygen uptake. BMC Pulm Med. 2010;10:31.

15. ATS Committee on Proficiency Standards for Clinical Pulmonary Function Laboratories. ATS statement: guidelines for the sixminute walk test. Am J Respir Crit Care Med. 2002;166:111-7.

16. Enright PL, Sherril DL. Reference equations for the sixminute walk in healthy adults. Am J Resp Crit Care Med. 1998;158:1384-7.

17. Troosters T, Gosselink R, Decramer M. Six minute walking distance in healthy elderly subjects. Eur Respir J. 1999;14:270-4.

18. Dourado VZ1. Reference equations for the 6-minute walk test in healthy individuals. Arq Bras Cardiol. 2011, pii: S0066$782 \times 2011005000024$

19. Araujo CO, Makdisse MR, Peres PA, Tebexreni AS, Ramos LR, Matsushita AM, et al. Different patterns for the 6-minute walk test as a test to measure exercise ability in elderly with and without clinically evident cardiopathy. Arq Bras Cardiol. 2006;86:198-205.
20. Britto RR, Probst VS, Dornelas de Andrade AF, Samora GAR, Hernandes NA, Marinho PEM, et al. Reference equations for the six-minute walk distance based on a Brazilian multicenter study. Braz J Phys Ther. 2013;17:556-63.

21. Iwama AM, Andrade GN, Shima P, Tanni SE, Godoy I, Dourado VZ. The six minute walk test and body weight-walk distance product in healthy Brazilian subjects. Braz J Med Biol Res. 2009;42:1080-5.

22. Osses AR, Yanez VJ, Barria PP, Palacios MS, Dreyse DJ, Díaz PO, et al. Reference values for the 6-minutes walking test in healthy subjects 20-80 years old. Rev Med Chil. 2010;138:1124-30.

23. Alameri H, Al-Majed S, Al-Howaikan A. Six-min walk test in a healthy adult Arab population. Respir Med. 2009;103: 1041-6.

24. Masmoudi K, Aouicha MS, Fki H, Dammak J, Zouari N. The six minute walk test: which predictive values to apply for Tunisian subjects aged between 40 and 80 years? Tunis Med. 2008;86:20-6.

25. Ben Saad H, Prefaut C, Tabka Z, Mtir AH, Chemit M, Hassaoune $\mathrm{R}$, et al. 6-minute walk distance in healthy North Africans older than 40 years: influence of parity. Respir Med. 2009;103:74-84.

26. Richalet JP. Altitude and the cardiovascular system. Presse Med. 2012;41 6 Pt 1:638-43.

27. Heredia P, Fonseca Z. Encuesta nacional de la situación nutricional Colombia 2010, Ministerio de la Protección Social e Instituto Colombiano de Bienestar Familiar, Componente Antropometría. Acceso 22 de Diciembre 2016. Disponble en: minsalud.gov.co/sites/rid/Lists/BibliotecaDigital/RIDE/VS/ED/ GCFI/Base\%20de\%20datos\%20ENSIN\%20-\%20Protocolo\%20 Ensin\%202010.pdf.

28. Carvalho VO. Aerobic exercise prescription in patients with chronic heart failure: a review in the beta-blocker era. J Cardiovasc Med (Hagerstown). 2012;13:570-4.

29. Casanova C, Celli BR, Barria P, Casas A, Cote C, de Torres JP, et al. The 6-min walk distance in healthy subjects: reference standards from seven countries. Eur Respir J. 2011;37:150-6.

30. Fiorina C, Vizzardi E, Lorusso R, Maggio M, De Cicco G, Nodari $\mathrm{S}$, et al. The 6-min walking test early after cardiac surgery. Reference values and the effects of rehabilitation programme. Eur J Cardiothorac Surg. 2007;32:724-9.

31. Lipkin DP, Scriven AJ, Crake T, Poole-Wilson PA. Six minute walking test for assessing exercise capacity in chronic heart failure. Br Med J (Clin Res Ed). 1986;292:653-5.

32. Salbach NM, O'Brien KK, Brooks D, Irvin E, Martino R, Takhar $P$, et al. Reference values for standardized tests of walking speed and distance: a systematic review. Gait Posture. 2015;41:341-60.

33. Chetta A, Zanini A, Pisi G, Aiello M, Tzani P, Neri M, et al. Reference values for the 6-min walk test in healthy subjects 20-50 years old. Respir Med. 2006;100:1573-8.

34. Tanaka H, Monahan K, Seals D. Age-predicted maximal heart rate revisited. J Am Coll Cardiol. 2001;37:153-6.

35. Baeza-Barría VC, San Martín-Correa MA, Rojas-Rojas GA, Martínez-Huenchullán SF. Respuesta fisiológica en el test de marcha en 6 minutos en pacientes con enfermedad pulmonar obstructiva crónica. Fisioterapia. 2014;36:160-6.

36. Langlois JA1, Keyl PM, Guralnik JM, Foley DJ, Marottoli RA, Wallace RB. Characteristics of older pedestrians who have difficulty crossing the street. Am J Public Health. 1997;87:393. 